(C) [2005] IEEE. Reprinted, with permission, from [Jill Alison Walker, David G. Dorrell and Calum Cossar, Fluxlinkage calculation in permanent-magnet motors using the frozen permeabilities method, Magnetics, IEEE

Transactions on Oct. 2005]. This material is posted here with permission of the IEEE. Such ermission of the IEEE does not in any way imply IEEE endorsement of any of the University of Technology, Sydney's products or services. Internal or personal use of this material is permitted. However, permission to reprint/republish this material for advertising or promotional purposes or for creating new collective works for resale or redistribution must be obtained from the IEEE by writing to pubs-permissions@ieee.org. By choosing to view this document, you agree to all provisions of the copyright laws protecting it 


\title{
Flux-Linkage Calculation in Permanent-Magnet Motors Using the Frozen Permeabilities Method
}

\author{
Jill Alison Walker, Student Member, IEEE, David G. Dorrell, Member, IEEE, and Calum Cossar
}

SPEED Laboratory, Department of Electronics and Electrical Engineering, University of Glasgow, Glasgow G12 8LT

\begin{abstract}
Finite-element analysis can be used to determine the magnetization characteristics in terms of curves of flux-linkage against current or rotor position. The "frozen permeabilities" technique is presented as a method of apportioning flux-linkage contributions to the phase currents and permanent magnets, and for inductance calculations. Results from a split-phase interior permanent magnet motor are presented and compared with experimental data. Drawbacks to the method are discussed.
\end{abstract}

Index Terms-Electric machines, finite-element (FE) calculations, permanent magnets, time-dependent magnetic fields.

\section{INTRODUCTION}

$\mathbf{I}$ N PERMANENT-MAGNET (PM) motors, the excitation is provided by two sources: the armature current, which can be removed, and the PMs, which provide a constant source of excitation. In the design process it can be desirable to calculate the individual flux contributions from the currents and the PMs. Methods such as the phasor diagram rely on superposition to extract the individual flux-linkage contributions, which are then used to calculate the direct and quadrature axis inductances. Such methods assume the magnet flux remains constant at the open-circuit value when in fact it varies according to load.

In finite-element (FE) simulations, it is possible to split the flux-linkage into individual components by freezing the permeabilities. Verification of the results by measurement is complicated by the constant excitation of the PMs. The method presented here uses a combination of static and dynamic tests to measure the flux-linkage contribution from the PMs. Frozen permeability simulation results from a commercial FE package are verified using the proposed measurement techniques.

\section{Frozen Permeability Method}

The frozen permeability technique is a method for separating the total flux-linkage into contributions from the current and PMs in FE simulations. The flux-linkage is determined from the magnetic vector potential. An initial nonlinear solution is calculated for the given rotor position and load current and the resulting permeabilities in each element of the FE mesh stored. Using these permeabilities, one field source can be "turned off" and a new linear solution calculated, to determine the flux-linkage contribution from the remaining source. For example, to calculate the flux-linkage due to the PMs, the phase currents would be set to zero. To calculate the flux-linkage contribution from the phase currents, the remanent flux of the PMs $(\mathrm{Br}, \mathrm{Bt})$ would be set to zero. The sum of the two individual components will equal the total flux-linkage as calculated in the original nonlinear solution. Flux plots corresponding to the separate frozen permeability solutions are shown in Figs. 1 and 2.

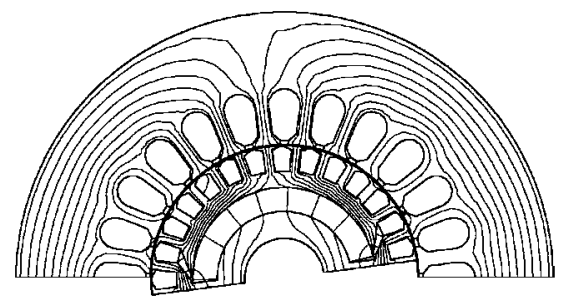

Fig. 1. Flux plot showing frozen permeability solution with currents only.

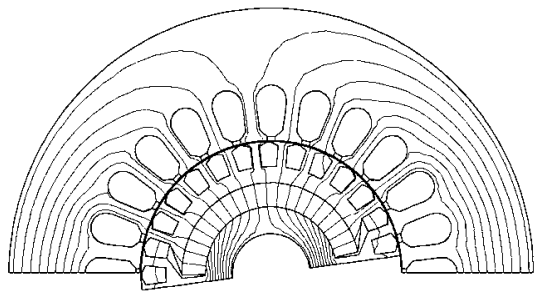

Fig. 2. Flux plot showing frozen permeability solution with magnets only.

The method was used by Bianchi et al. to calculate the parameters of a PM synchronous motor [1] and is included as a feature in a number of software packages [2], [3]. There is little mention of the method in literature and, in particular, there is a lack of experimental data to substantiate whether the method is in fact valid. Although the sum of the individual contributions equals the total flux-linkage from the nonlinear solution, the weighting of the flux-linkage due to current and magnet flux-linkage contributions may be wrong. Without experimental verification, there is no way of knowing if the solutions determined by the frozen permeability method are correct. The flux-linkage due to the PMs can be measured on open-circuit, but there is no way of measuring it directly under load conditions. The magnet flux-linkage must, therefore, be found by subtraction of the current contribution from the total flux-linkage, taken from the motor magnetization curves.

\section{Measurement of Magnetization CuRves}

The magnetization curves for the motor are presented in the form of flux-linkage versus current for successive rotor positions. Previously the magnetization curves may have been constructed by adding the flux-linkage due to current (as calculated from the locked-rotor tests) to the value of open-circuit magnet 


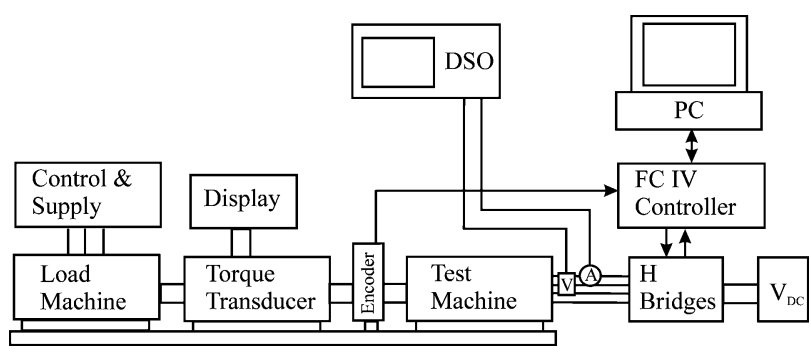

Fig. 3. Dynamometer test setup.

flux-linkage at the given rotor position [determined by integration of the open-circuit back electromotive force (EMF) waveform]. This method cannot be considered accurate as it assumes the magnet flux remains constant at the open-circuit value.

A dynamic test is required to measure the total flux-linkage under load conditions. To include the flux-linkage contributions from the PMs, the rotor must be rotated so that the magnets pass under the phase winding. With constant current in the phase winding, it is possible to determine the change in total fluxlinkage between successive rotor positions, but not the absolute value of flux-linkage at each position. To determine the absolute value and measure the magnetization curves, it is necessary to have a reference point whereby at one rotor position the total flux-linkage is already known. If there was no cross-saturation of the magnetic flux paths, no flux-linkage contribution from the PMs would be seen on the quadrature axis, but to assume this is no more valid than the assumption that the magnet flux is independent of load.

The solution is to calculate the total flux-linkage from the $i-\psi$ (ipsi) loop. Detailed information can be found in [4], [5]. The $i-\psi$ loop is used to determine the average electromagnetic torque from the change in coenergy over one electromagnetic cycle. The change in coenergy is equal to the area enclosed by the current versus flux-linkage trajectory. Because the current used to drive the motor is periodic, and for the brushless synchronous motor is sinusoidal, for every revolution there will be two points where the phase current is zero. The flux-linkage on open-circuit can be calculated from the back EMF waveform, and so the locus of the ipsi loop can be calculated with respect to the two open-circuit points. If rotor position sensing is included in the ipsi loop measurement, the total flux-linkage at each rotor position can be determined. The dynamometer setup is shown in Fig. 3.

\section{EXPERIMENTAL RESULTS}

Results are presented for a split-phase, two-pole interior PM motor with sinusoidally distributed motor winding, Fig. 2. For each rotational test, the phase voltage, phase current, and position information are stored. The data is then manipulated using MATLAB [7] into tables of total flux-linkage versus current at each rotor position. Fig. 4 shows the magnetization curves for the main phase of the motor. The measured curves are represented by solid lines; dashed lines represent total flux-linkages calculated in FE from the magnetic vector potential. The direct and quadrature axis curves are labeled for reference. The measured flux-linkage contributions from the currents and the PMs

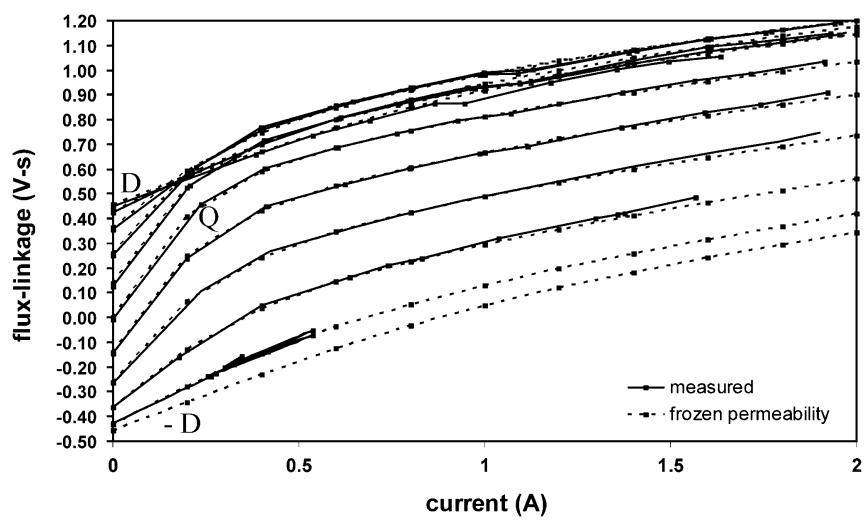

Fig. 4. Magnetization curves for main phase of test motor.

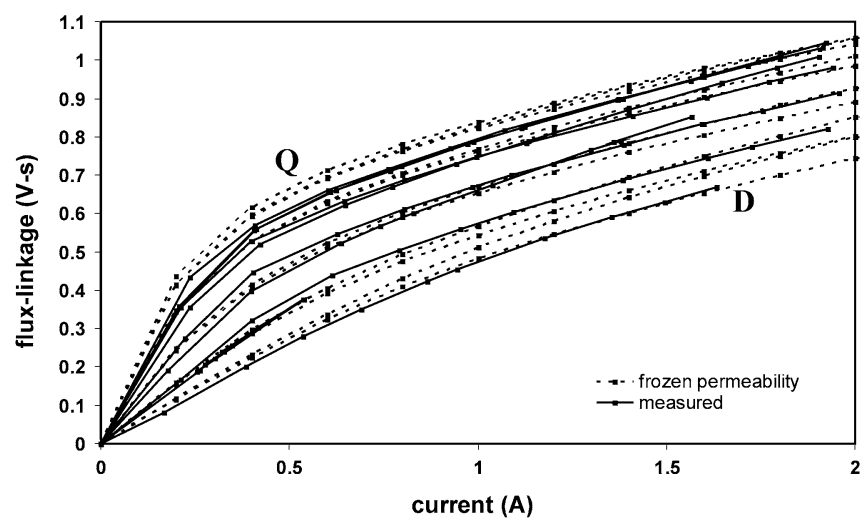

Fig. 5. Flux-linkage contribution from phase current.

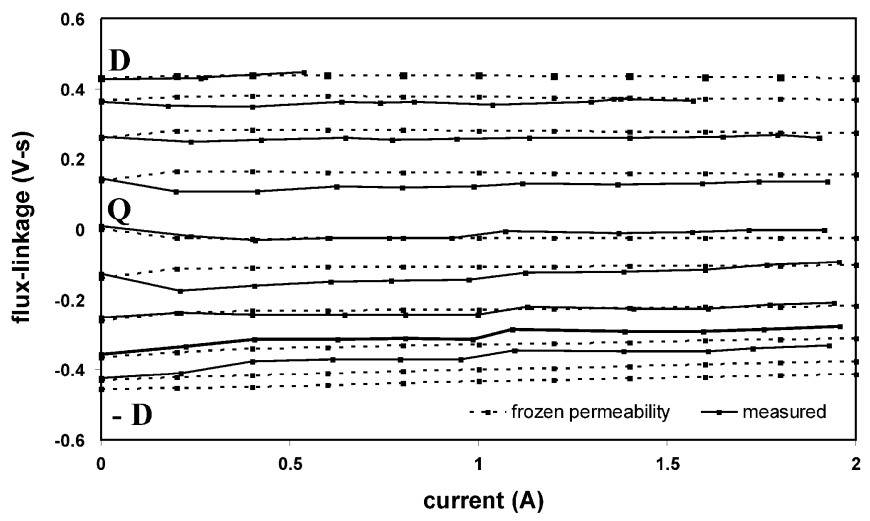

Fig. 6. Flux-linkage contribution from PMs.

are shown in Figs. 5 and 6, respectively, along with results from the two-dimensional FE simulations.

\section{DISCUSSION}

The dynamic tests used to calculate the $i-\psi$ loops were originally driven with sinusoidal current, producing elliptical ipsi loops. However, a number of problems arise from using a sinusoidal current waveform. The sinusoidal variation in current means that to achieve the required current levels at each rotor position, the motor must either be run at currents higher than the rated currents, or with large torque angles $\gamma$. Care must be 
taken when selecting the range of $\gamma$, as high torque angles can lead to demagnetization of the embedded ferrite magnets.

A further consequence of running the motor with sinusoidal currents at different torque angles is that the resulting ipsi loops show there is not one unique magnetization curve for each rotor position; the curves are dependent on both the direction and rate of change of current. The effects are less prominent on the direct axis than on the quadrature axis, due to presaturation of the magnetic circuit by the PMs. Running the motor with square wave rather than sinusoidal currents reduces the range of gamma values required and also limits the rate-of-change effects. The resulting magnetization curves are single-valued and equivalent to static magnetization curves. The single-valued magnetization curves correlate well with the results from the nonlinear FE simulations. The magnetization curves generated from the sinusoidal $i-\psi$ loops showed considerable hysteresis effects, which are neglected in the FE simulations.

Although the two sets of magnetization curves show close correlation, there are discrepancies between the measured current and magnet flux-linkage contributions and those calculated from the frozen permeabilities method. However, both sets of results show that the PM flux-linkage varies with load conditions.

Variation between the individual components may be due in part to the representation of the motor materials in the FE software. The magnet information was taken from manufacturers' data sheets. Under the sinusoidal ipsi loop test conditions, the ferrite magnets were demagnetised slightly. Although the remanent flux $B_{r}$ has been adjusted accordingly, the demagnetization may not be uniform across the magnet cross-section. For the purposes of the simulations, the magnets are assumed to have a remanent flux of 0.32 and a recoil permeability of 1.08. The rotor and stator laminations have a complicated geometry and although the materials are represented by measured B-H curves, the magnetic properties will vary from a single sheet tester sample, due to rotational fluxes and the effects of punching and stamping. Slight changes in the material data were found to have an effect on the separation into individual flux components by the proposed method. A number of possible combinations of steel B-H curve and magnet data may exist which correlate well with the measured magnetization curves, but provide different results when the separation of the fluxes by the frozen permeability method is carried out.

The difficulty in verification of the method arises because we cannot measure the exact quantities that are calculated in the frozen permeability method. The locked-rotor tests measure a change in flux-linkage due to an applied current, but we cannot measure the magnet flux-linkage under this applied current directly. Although a value for the magnet flux can be derived, it makes use of the principle of superposition. The inherent nonlinearity of the system cannot be ignored. The separation into individual components by the FE software is linear, but the permeabilities used in the solution are nonlinear. Accurate measurement of the different flux components, as calculated in the FE analysis, does seem to be an intractable problem.

\section{CONCLUSION}

The frozen permeability method has been discussed as a means of determining the proportion of total flux-linkage attributable to the PMs and phase currents. A method of measuring the flux components, as a means of verifying the frozen permeability results, has been suggested. The difficulties in measuring the individual components in the nonlinear system are acknowledged. Although the results from nonlinear simulations correlate well with the measured data, there are discrepancies in the frozen permeability results. Further work is needed to determine the sensitivity of the method to the material data used in the simulations.

\section{ACKNOWLEDGMENT}

This work was supported in part by the SPEED Consortium, in part by the U.K. Engineering and Physical Sciences Research Council, and in part by Robert Bosch GmbH. Test motors were provided by Electrolux Compressors, Italy. The authors thank W. MacDougall and J. Kelly for construction of the test rigs. They also thank Dr. M. Popescu for help with the PC-FEA scripting.

\section{REFERENCES}

[1] N. Bianchi and S. Bolognani, "Magnetic models of saturated interior permanent magnet motors based on finite element analysis," in Proc. 33rd IAS Annu. Meeting, vol. 1, Oct. 12-15, 1998, pp. 27-34.

[2] Ansoft Corporation, Maxwell 2D.

[3] SPEED Software Suite, Speed Laboratory, Univ. Glasgow, Glasgow, Scotland.

[4] M. R. Harris and T. J. E. Miller, "Comparison of design and performance parameters in switched reluctance and inductance motors," in Proc. 4th Int. Conf. Elect. Mach. Drives, Sep. 13-15, 1989, pp. 303-307.

[5] D. Staton, W. Soong, C. Cossar, and T. J. E. Miller, "The unified theory of torque production in switched and synchronous reluctance motors," in Proc. 6th Int. Conf. Elect. Mach. Drives, Sep. 8-10, 1993, pp. 67-72.

[6] T. J. E. Miller, J. A. Walker, and C. Cossar, "Measurement and application of flux-linkage in a permanent magnet synchronous motor," in Proc. 2nd Int. Conf. Power Electron., Mach. Drives, vol. 2, Mar. 31-Apr. 2 2004, pp. 674-678.

[7] Matlab, The Mathworks, Inc. 